\title{
4D Printing Inflatable Silicone Structures
}

\author{
Fergal B. Coulter and Anton lanakiev
}

\begin{abstract}
This article details steps in creating low-power artificial muscles using 3D printing. It describes a manufacturing system that can be used to fabricate seamless tubular dielectric elastomer actuators (DEA), for eventual use in biorobotic devices. The focus is on producing passive elastomeric components of DEA and the dimensional changes that occur after printing is complete. A four-axis printing system is described, capable of spray depositing multilayer tubular silicone membranes onto an air-permeable mandrel. Mechanical strain was imparted in the membranes by means of inflation. A laser measurement system was constructed to act as a 3D scanner, which measured the shape of the inflated "balloon." The surface shape was reconstructed in software using the parametric modeling tool Grasshopper. Seamless auxetic tessellations were calculated across the entire surface, and then converted to CNC GCode. These toolpaths were then physically extruded over the surface of the balloon, stacked five layers high. When the extruded silicone structure was completely cured, the pneumatic strain was released, allowing the structure to collapse evenly. The compression in the printed structure was balanced by the tension in the stretched membranes, thus producing a minimum energy structure.
\end{abstract}

\section{Background}

Dielectric ELASTOMER ACTUATORS (DEA) are electromechanical transducers. Actuation of a DEA system is achieved via compliant (stretchable) electrodes applied on either side of a thin elastomer membrane. They are essentially flexible capacitors. An applied voltage (kV range) will generate electrostrictive attraction (coulombs force) between the two electrodes, resulting in a compression and equibiaxial elongation of the dielectric membrane. ${ }^{1}$

Prestretching the membrane increases the surface area while reducing the thickness. The result is stored strain energy in the elastomer. Electrical efficiency is increased by reducing required activation voltages, and resistance to electric breakdown is improved. ${ }^{2,3}$ The elastic strain energy will have a tendency to return the elastomer to its unstretched state if not constrained.

Using pneumatic inflation to maintain the stretch is described in ref. ${ }^{4}$ Bonding a flexible but incompressible frame to the stretched elastomer is discussed in ref., ${ }^{5}$ where the mechanical strain and compressive resistance equalize by buckling out of plane to become a minimum energy structure.

This research combines these two concepts along with the creation of spray-deposited silicone membranes, as per ref. ${ }^{6}$ Prestrained by inflation, minimum energy structures are created by extruding a semirigid silicone over the surface of the inflated balloon, creating a reciprocal framelike support structure. The frames created are in the auxetic "hexachiral" class of geometries. ${ }^{7}$ Their purpose in this case is to constrain the inflated DEA membranes in an even manner, without succumbing to nonradial or out of plane buckling.

\section{Materials and Methods}

A system was built to spray-deposit multiple thin (tens of $\mu \mathrm{m}$ ) layers of low-hardness (Smooth-On Ecoflex 00-30) silicone rubber in a repeatable manner onto a rotating cigarshaped air-permeable mandrel. Even layer thickness deposition was aided by reducing material viscosity with an aliphatic hydrocarbon solvent (Arrow Lotoxane). Viscosity was brought from $6000 \mathrm{mPa} \cdot \mathrm{s}$ down to below $3000 \mathrm{mPa} \cdot \mathrm{s}$ when $10 \%$ solvent was added. The material was atomized using a spray valve (Techcon TS5540) with a $1.17 \mathrm{~mm}$ round nozzle.

A cigar-shaped mandrel was mounted horizontally, attached to a stepper motor on one end. The other end contained a push-fit pneumatic fitting attached to a steel pipe that extended to the air-permeable region at the center of the mandrel. A schematic of the spray system is shown in Figure 1a.

Multiple silicone layers were deposited by rotating the mandrel at a fixed speed (200 RPM) while moving the spray nozzle parallel to the mandrel axis, at a constant distance

School of Architecture, Design and Built Environment, Nottingham Trent University, Nottingham, United Kingdom. 


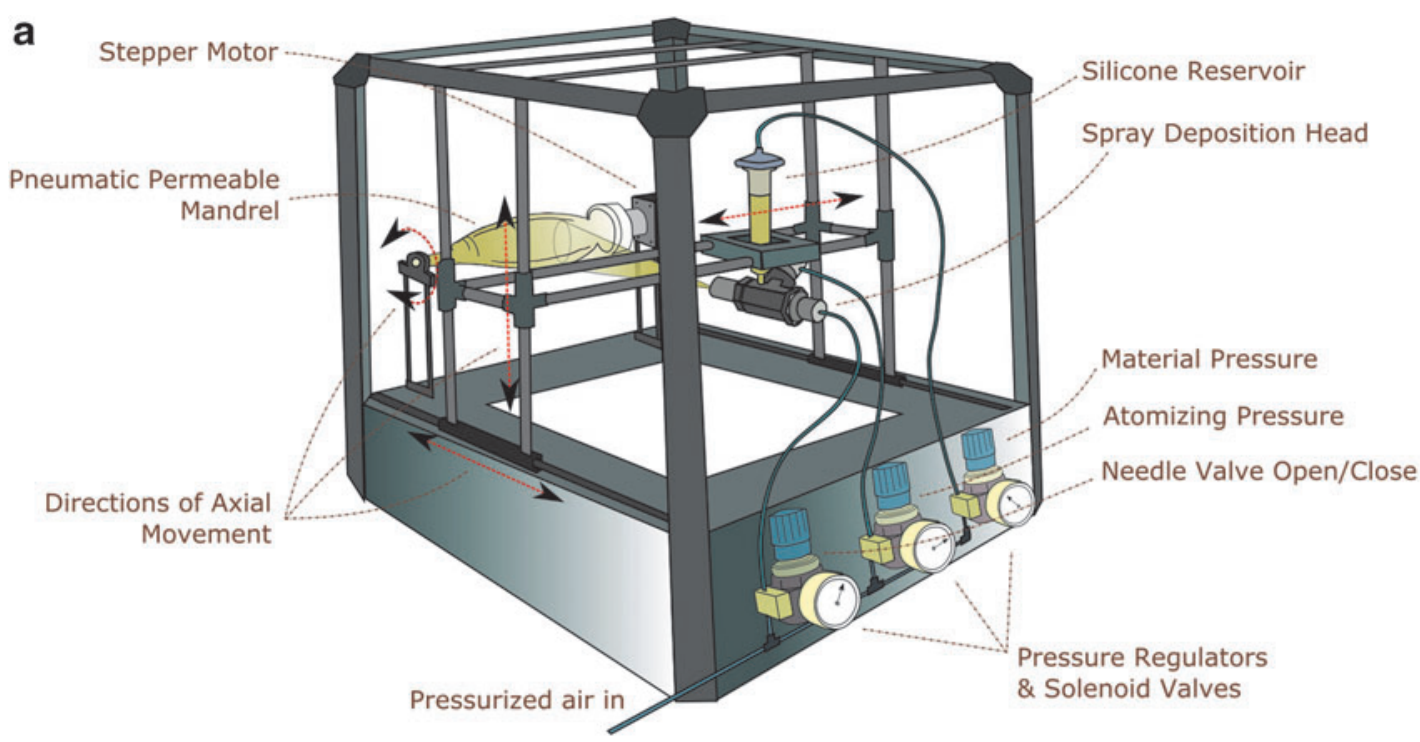

b

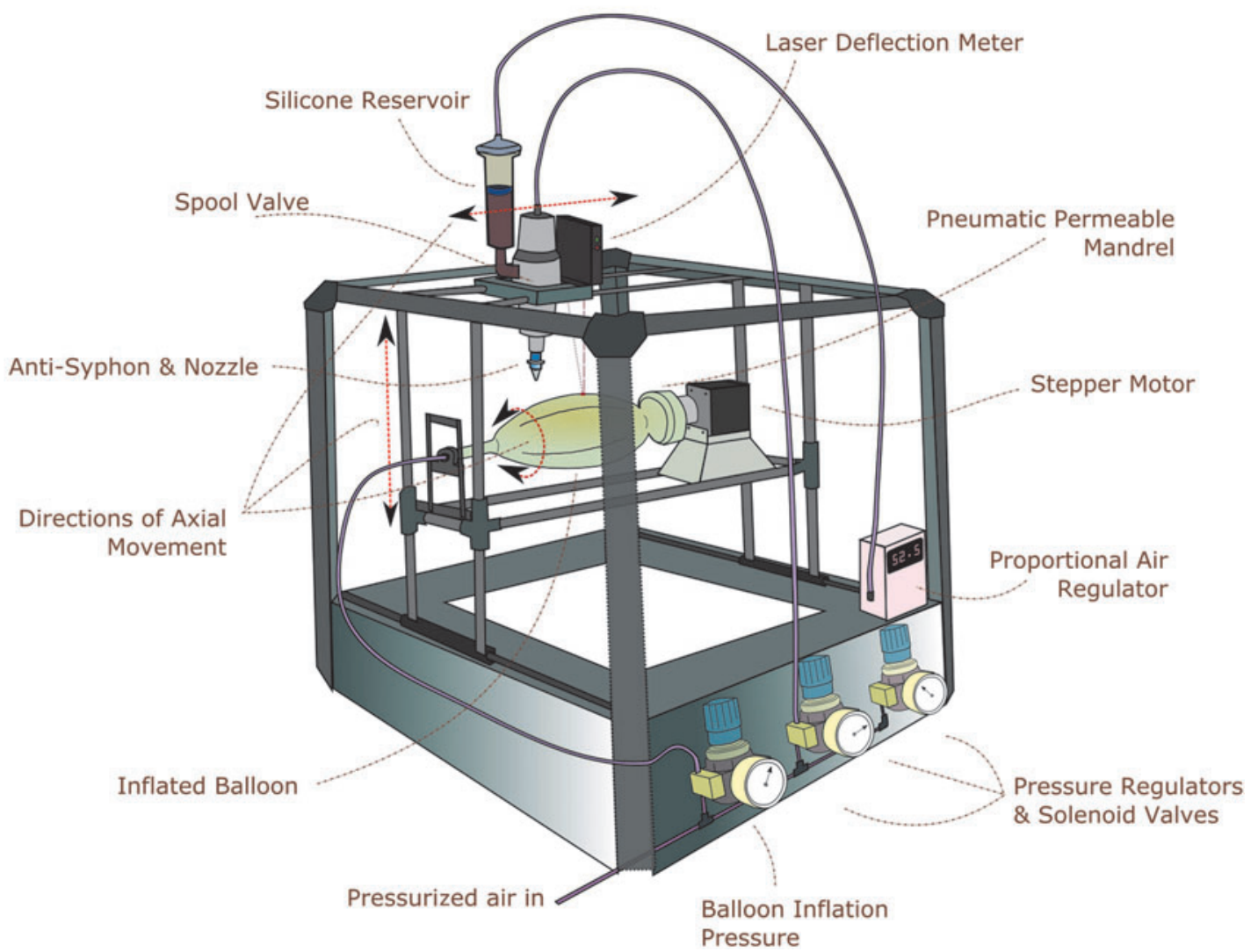

FIG. 1. (a) Schematic of 3D printer in spray configuration. (b) Printer in can and deposition configuration.

from the substrate. A constant angular velocity (CAV) strategy was employed to improve the evenness of each layer, irrespective of the changing surface linear velocity of the substrate. To achieve CAV, the spray carriage was moved at a speed inversely proportional to the mandrel radius at that particular spray focal point. The end result was that the spray head would spend the same amount of time over any particular area of mandrel surface, regardless of the circumference at that particular cross section. The curing of each layer was accelerated by the manual introduction of a $500 \mathrm{~W}$ infrared heat lamp for 2 minutes before removal. An example of the cross-sectional thickness for each layer is shown in Figure 2. Each layer was coated by brushing the surface with graphite. This can act as a DEA electrode. Microscopic examination showed that the inner six layers measured on average $82 \mu \mathrm{m}$ thick with a standard error of $3.74 \mu \mathrm{m}$.

Regression analysis showed that the variables with the most influence on layer thickness were carriage speed, 


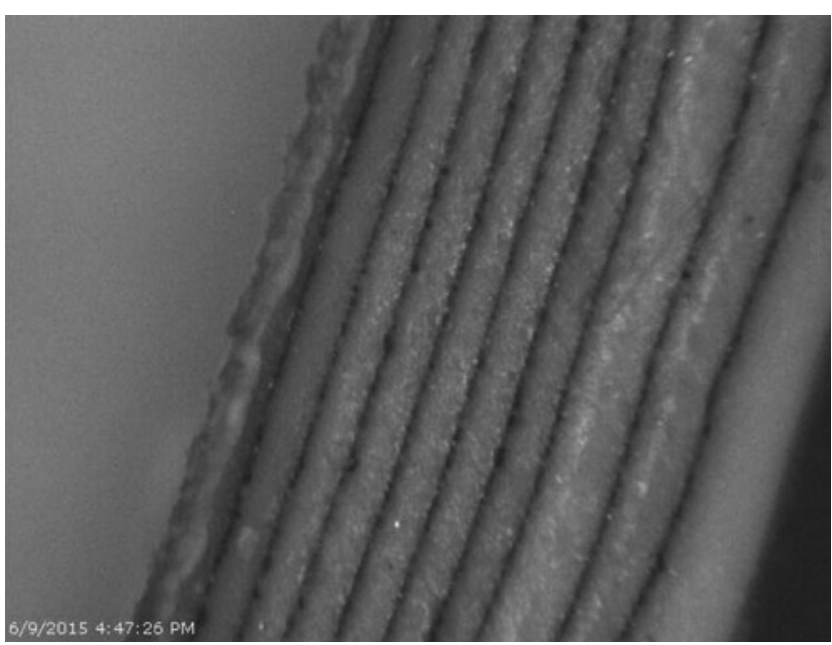

FIG. 2. Cross section of sprayed layers. The leftmost layers are deliniated by graphite powder after depositing a single layer; the rightmost are double-sprayed layers.

material pressure, and solvent quantity. In these tests, the optimum (thinnest) repeatable layer was $40 \mu \mathrm{m}$. This was achieved by moving the print head at a maximum speed of $3200 \mathrm{~mm} / \mathrm{min}$, a material and atomizing pressure of $3 \mathrm{bar}$, and $150 \mathrm{~mm}$ between substrate and spray head. An example of a preinflated mandrel is shown in Figure 3.

Mechanical prestrain was imparted by pneumatic inflation, where a volume of compressed air was passed through the mandrel from its core to outer surface. Following inflation, the shape of the inflated structure was digitally measured using a laser deflection meter (Banner LG10). This was mounted in-line with the deposition nozzle on a customized $3 \mathrm{D}$ printer. The laser was programmed to act as a 3D scanner, measuring 12 concentric rings on the inflated balloon, at prescribed points along the axis. Difficulties arose in measurement due to elastomer transparency and specular reflection, but were overcome using silicone pigment and dusting the surface with talc, respectively. A digital surface repre-

F4 sentation (Figure 4a) was generated from the measurements within the CAD software Rhino3D and parametric design plugin Grasshopper.

This digital surface was used to calculate toolpaths for various auxetic geometries based in the hexachiral honeycomb class-an example of which is shown in Figure 4b. The radius and count (both longitudinally and circumferentially)

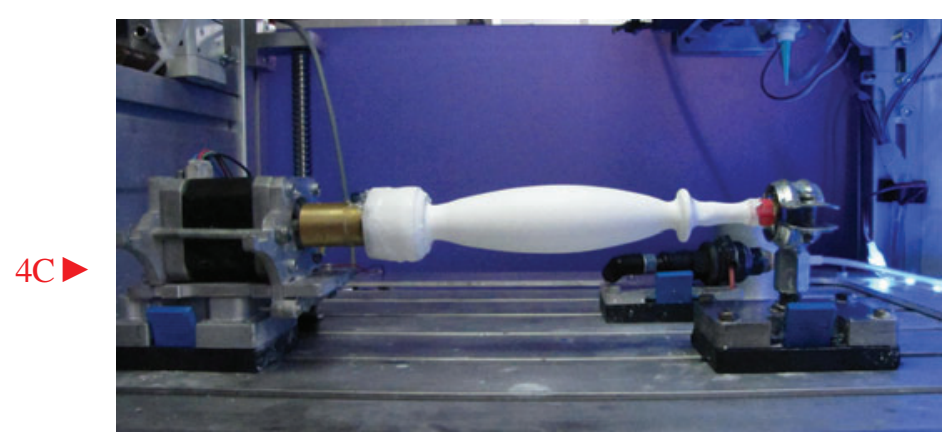

FIG. 3. Mandrel spray-coated with silicone, before inflation. of the nodes, along with the tangent angle and chirality of the ligaments, were all parametrically adjustable. As discussed in the Background section, the purpose of these toolpaths was to print a collapsible outer support lattice, capable of constraining some (but not all) of the mechanical prestrain in the elastomer layers, following the removal of internal pneumatic pressure.

Hexachiral geometries were chosen based on their ability to compress equibiaxially without resorting to surface buckling, thus maintaining a smooth curved balloon-like surface. Chiral structures are noted for being tunable in their resistance to compressive loading, depending on their ligament length, count, and tangency.

The toolpaths were converted to GCode within the Grasshopper environment. The GCode was created by "unrolling" the wrapped pseudo-cylindrical geometry from polar-cylindrical to Cartesian coordinates. This process is somewhat analogous to Mercator projection in cartography. Figure $4 \mathrm{c}$ shows the result of this process. The calculated toolpaths were extruded and bonded on to the surface of the inflated membranes by means of a customized four-axis printer. The print material was a hard but flexible Shore 73A (Thomson Bros. Thomtastic 73) silicone, made thixotropic with $10 \%$ kaolin mineral filler and $5 \%$ alumina trihydrate.

The printer used was based on a Leapfrog "Creatr" printer. This is a standard XYZ (H-gantry) FDM printer, with hotend removed and replaced with a high-viscosity spool valve (Techcon TS-941). A 23-gauge, $0.56 \mathrm{~mm}$ precision dispensing nozzle (Fisnar Micron-S) was found to work best, balancing fast reaction times to valve on/off events, a thin output bead and low susceptibility to clogging. The system was calibrated before each print run commenced, ensuring that the laser scanner measurements and extrusion nozzle tip were in sync with each other.

To maintain a constant bead thickness over the surface of the inflated membrane, the print head speed was varied continuously throughout the print. The calculated speed varied with the circumference of the substrate at each point, together with the angle of the extruded line in relation to the $y$ axis. It was essential to decrease the head speed in proportion to any increase in $z$ height of substrate due to the variation in surface linear velocity of the rotating substrate. The speed required to maintain a constant substrate linear velocity was calculated in Grasshopper. The net effect was that the printer extruded at a constant $800 \mathrm{~mm} / \mathrm{min}$. Figure 5 shows an example of a printed hexachiral structure, before internal air pressure was removed.

Aligning and positioning the stacked layers was achieved by outwardly offsetting the computed surface for each layer and then recalculating the desired chiral structure. When printing was complete, the support structure was left overnight before being vulcanized by heat acceleration. This time period was found to significantly improve the bond between balloon and printed structure. The internal pressure was then removed so that it no longer played any part in stretching the membrane. Any strain maintained is a product of the support frame alone, and so the system can be considered a minimum energy structure. Figure 6 shows the two states of one such printed structure. The structures can be thought of as 4D prints because they change shape following the removal of the air pressure, but retain the ability to return to their original form. 

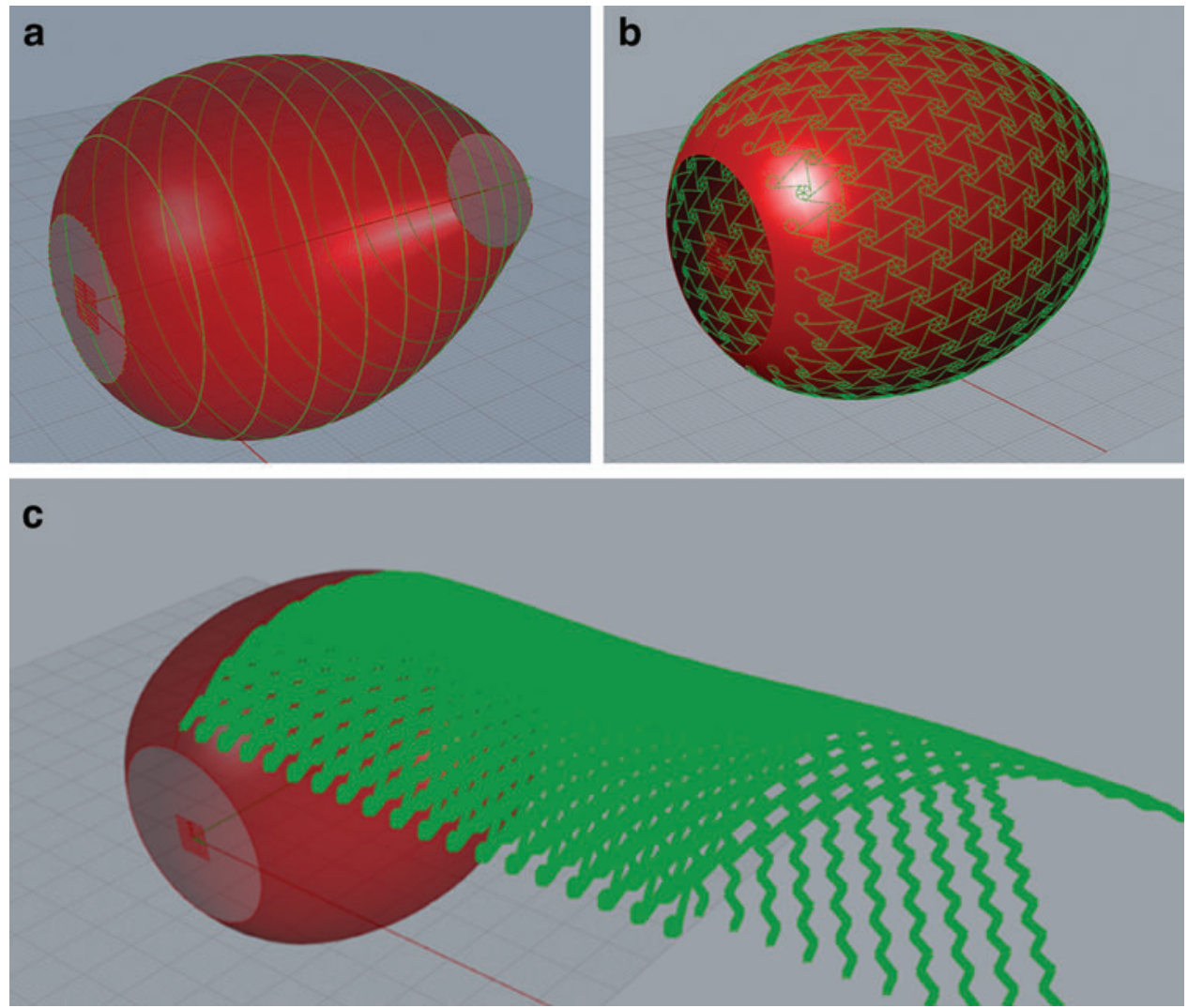

FIG. 4. (a) Surface created by lofting scanned concentric rings. (b) Calculated hexachiral toolpaths. (c) Conversion to Cartesian coordinates.

\section{Conclusions}

Various prints were completed, each with different chiral geometries and printed wall thicknesses. All displayed a unique ability to constrain the tensioned membrane and come to rest at different minimum energy configurations.

Limitations of the four-axis system were realized when printing on a substrate with a high degree of curvature. The offset toolpaths were stacked such that walls and nodes were normal to the surface, while the extrusion tip was always perpendicular to the mandrel axis. After $3 \mathrm{~mm} z$ height, this generally resulted in gravity pulling and distorting the

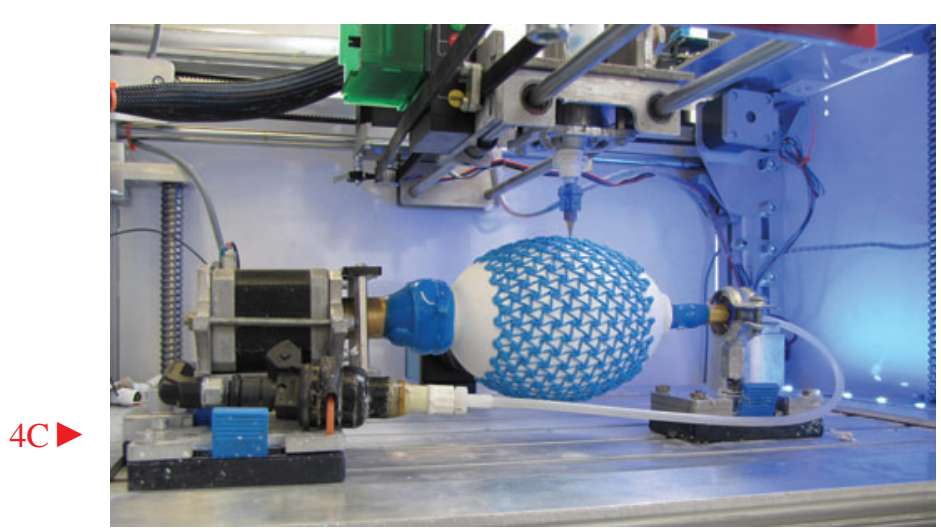

FIG. 5. Hexachiral structure printed on inflated balloon Also visible are mandrel, motor and tailstock assembly, material and inflation valve, and laser scanner. extruded walls, thus preventing further layers from being deposited accurately.

Nonetheless, this work represents a promising first step in 4D printing tubular, fully materially compliant, dielectric elastomer minimum energy structures for soft robotic systems. More examples of these structures are displayed in ref. ${ }^{8}$
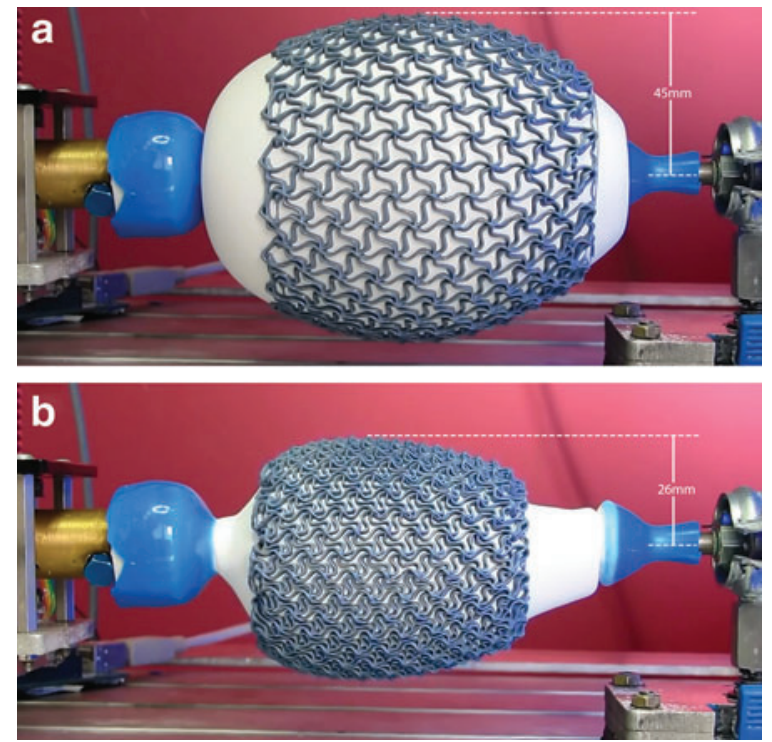

FIG. 6. (a) Fully inflated balloon with 3-mm-high printed chiral structure. (b) Structure with air pressure removed, in minimum energy configuration. 


\section{PRINTING INFLATABLE STRUCTURES}

\section{References}

1. Kofod G. Dielectric elastomer actuators. PhD Thesis, The Technical University of Denmark, 2001.

2. Kofod G. The static actuation of dielectric elastomer actuators: How does pre-stretch improve actuation. J Phys D Appl Phys 2008;41:215405.

3. Plante JS, Dubowsky S. Large-scale failure modes of dielectric elastomer actuators. Int J Solids Struct 2006;43: 25-26.

4. Soleimani M, Menon C. Preliminary investigation of a balloon shaped actuator based on electroactive elastomers. Smart Mater Struct 2010;19:047001.

5. Petralia MT, Wood R. Fabrication and analysis of dielectricelastomer minimum-energy structures for highly-deformable soft robotic systems. 2010 IEEE/RSJ International Conference on Intelligent Robots and Systems (IROS), 2010.
6. Araromi OA, Conn AT, Ling CS, et al. Spray deposited multi-layered dielectric elastomer actuators. Sens Actuat A 2011;167:459-467.

7. Rossiter J, Takashima K, Scarpa F, et al. Shape memory polymer hexachiral auxetic structures with tunable stiffness. Smart Mater Struct 2014;23:045007.

8. Coulter FB. 2015. Personal website: www.fergalcoutler.eu

Address correspondence to: Fergal B. Coulter School of Architecture, Design and Built Environment Nottingham Trent University Burton Street Nottingham NG1 4BU United Kingdom

E-mail: fergal.coulter@gmail.com 


\section{AUTHOR QUERY FOR 3DP-2015-0017-VER9-COULTER_1P}

AU1: Address of correspondence correct? If not, please amend as needed. 